\title{
Diverticular disease
}

David Humes

Robin Spiller

David Humes BSc MSc MB BS PhD FRCS is an Associate Professor of Surgical Epidemiology/Honorary Colorectal Surgeon NIHR Nottingham Biomedical Research Centre, Nottingham University Hospitals NHS Trust and University of Nottingham, and Nottingham Digestive Diseases Centre, School of Medicine, University of Nottingham, UK. His main research interest is the epidemiology of surgical disease, with a focus on diverticular disease. Competing interests: none declared.

Robin Spiller MB BChir MSc MD FRCP is a Professor of Gastroenterology NIHR Nottingham Biomedical Research Centre, Nottingham University Hospitals NHS Trust and University of Nottingham, and Nottingham Digestive Diseases Centre, School of Medicine, University of Nottingham, UK. His main research interests are functional gastrointestinal diseases, especially postinfectious irritable bowel syndrome and symptoms after acute diverticulitis. Competing interests: none declared.

\begin{abstract}
Colonic diverticula are formed by mucosal outpouching from the colonic wall. Their presence increases with increasing age with most, in Caucasians, being situated in the left colon. The aetiology of these outpouchings is not fully understood but dietary, lifestyle and genetic factors have all been implicated. Most patients with diverticula are asymptomatic (diverticulosis); however, approximately $20 \%$ develop symptoms such as intermittent abdominal pain and change in bowel habit and are said to have symptomatic diverticular disease. Acute diverticulitis is characterized by acute inflammation within these pockets, with associated constitutional symptoms. Other complications include perforation, abscess, fistulae, strictures and bleeding, albeit in relatively few patients. Stratifying patients using high-quality CT is important as it allows a more tailored approach to treatment. Managing chronic symptoms in symptomatic diverticulosis requires identifying those with longstanding irritable bowel syndrome, who might respond differently, from those with symptoms after acute diverticulitis. Planned surgical resection in symptomatic patients must be undertaken on a case by case basis. A high-fibre diet, cyclical antibiotics and anti-inflammatory treatments have been proposed, but no treatments have yet been shown to benefit unselected patients with symptomatic diverticular disease.
\end{abstract}

Keywords

Ageing; colon; diverticulitis; diverticulosis; inflammation

\section{Key points}

- Colonic diverticulosis is the presence of mucosal outpouchings from the colonic wall which becomes increasingly common with age.

- The majority of patients with colonic diverticulosis remain asymptomatic.

- The most common complications are acute diverticulitis, perforation, abscess formation, stricture, bleeding and fistula.

- In those presenting acutely with suspected complications an early CT scan helps risk stratify patients into those with uncomplicated and complicated disease and helps direct relevant treatment.

- Patients with CT proven uncomplicated acute diverticulitis may not require treatment with antibiotics. 


\section{Prevalence}

Diverticulosis is the presence of mucosal outpouchings through the wall of the colon. It is a common feature of ageing in the developed world, being rare below the age of 40 years and steadily increasing thereafter: $30 \%$ of individuals have it by 60 years and $50 \%$ by 75 years.

Fortunately, diverticulosis only causes symptoms in only around 1 in 5 individuals, remaining asymptomatic in the rest. Data from Swedish national medical records show that $40 \%$ of those aged $>60$ years have diverticulosis (400 in 1000); however, symptoms severe enough to necessitate hospitalization occur in only 1 in 1000 per year, giving a lifetime risk (assuming a span of 90 years) of $<1 \%$. This translates into around 200 patients with diverticulosis in an average general practice of 2000 patients, of whom perhaps 40 have symptoms but $<1$ requires hospitalization in any one year.

There are also important racial differences, with a striking predominance of right-sided diverticulosis in Japanese and South-Eastern Asian populations, compared with a left-sided predominance in Caucasians. Environmental factors play an important part, and migrants from lowrisk areas of the world gradually, over a 20-year period, acquire the prevalence typical of their new environment.

\section{Definition}

It is important to distinguish the presence of diverticula (asymptomatic diverticulosis) from symptomatic diverticular disease where diverticulosis is accompanied by various symptoms including abdominal pain, bloating and erratic bowel habit with both diarrhoea and constipation. Symptomatic diverticular disease manifests itself as a spectrum. It is believed that hard pellets of stool can form within a diverticulum and cause acute diverticulitis (Figure 1), resulting in micro-abscess formation. This can resolve spontaneously or progress to a state known as complicated diverticular disease, which can include abscesses, along with other complications such as colonic perforation, structuring, bleeding and fistulae (most commonly colo-vesical or colo-vaginal).

\section{Pathology and pathogenesis}

In diverticulosis, the mucosa prolapses through points of weakness in the colonic wall, usually where the mesenteric blood vessels pass through the muscle. Colonic resection specimens from patients with symptomatic diverticular disease show hypertrophy and disorganization of the circular smooth muscle with elastosis of the longitudinal muscle. There is an associated concertina effect, which can be seen endoscopically as prolapsing mucosal folds and narrowing of the lumen.

Diverticula are essentially mucosa with no supporting muscle and hence represent points of weakness. These become inflamed in diverticulitis, often associated with plugs of inspissated faecal material. Exactly how this causes mucosal breaks and allows access of bacterial products into the lamina propria, with a subsequent inflammatory response, is unclear but might be related to local changes in the microbiota. Abnormalities of enteric nerves with changes in neurochemical coding have been reported, but whether these are a response to inflammation or degenerative ageing changes in unclear. There are clearly important genetic factors, and heritability is estimated at around $40 \%$.

The role of diet is controversial, but the Oxford cohort of the European Prospective Investigation into Cancer and Nutrition (EPIC) study showed a protective effect of vegetarianism and adverse effects of smoking, obesity, hypertension and hyperlipidaemia. The North American prospective cohort study of 47,228 male health professionals (40-75 years old) who were free of diverticular disease at baseline in 1986 also showed decreased risk with increasing fibre intake, particularly fibre from vegetables and fruit, and an increased risk associated with eating red meat. A subsequent analysis also reported that lower levels of exercise and increasing obesity were risk factor for developing symptomatic diverticular disease. The fact that levels of obesity are steadily rising could account for some of the increased incidence of hospitalization for diverticular disease seen over the last decades even after adjusting for the effect of an ageing population.

\section{Course of the disease}

Although most people with diverticulosis are asymptomatic, approximately $20 \%$ report recurrent pain. The proportion varies in different patient cohorts depending on whether they are being investigated for symptoms (around one-third reporting chronic pain) or are undergoing colorectal cancer screening (a pain incidence of around 1 in 8 ). After a bout of acute diverticulitis, they are around four times more likely to experience recurrent abdominal pain and disturbed bowel habit. Other important risk factors for having symptoms include female gender, somatization and social isolation; these are similar to what is seen in IBS without diverticulosis and might reflect similar underlying mechanisms, including visceral hypersensitivity. Population studies suggest that loose, frequent stools are more likely than 
constipation, although whether this reflects patients' adopting the commonly advised high-fibre diet after being diagnosed with diverticular disease is unclear.

Of those admitted with acute pain, most are rapidly discharged with a diagnosis of uncomplicated acute diverticulitis. However, a few develop complicated disease that can require emergency surgery to treat abscesses, perforation, fistulae and strictures.

\section{Diagnosis}

Individuals with uncomplicated diverticular disease experience symptoms similar to those of IBS, with recurrent abdominal pain in bouts lasting a few hours associated with an erratic bowel habit. However, those with acute diverticulitis have prolonged pain that is well localized, usually to the left iliac fossa, and often associated with guarding and rebound tenderness. Such pain is typically associated with fever and systemic features such as anorexia and lethargy. Individuals with perforation can present with a sudden onset of severe abdominal pain, which can be localized to the left iliac fossa or widespread, associated with localized or generalized peritonitis. Patients with an abscess can present with localized or generalized pain with an associated swinging fever. Symptoms of a diverticular fistula depend on the site of fistula formation but can include pneumaturia (bubbles in the urine) and recurrent urinary tract infections with a colovesical fistula, or passage of faeces via the vagina in a colovaginal fistula.

\section{Investigations}

Most patient with diverticulosis are diagnosed after investigation with either endoscopy or CT in which diverticulosis is an incidental finding. Full blood count, urea and electrolytes, C-reactive protein (CRP) and urine analysis are useful, followed in most cases by CT in those presenting acutely to hospital. Blood tests can show an elevated white blood count and CRP concentration. CT shows a thickened bowel wall with fat-stranding with or without local abscess formation or perforation (Figure 1). Early CT helps to risk-stratify patients with acute diverticulitis into those with and without complications, which can help direct treatment, including appropriate use of antibiotics.

\section{Differential diagnosis}

The differential diagnosis of diverticular disease is outlined in Table 1.

\section{Treatment}

No treatment is required for individuals with diverticulosis. Those with diverticular disease may benefit from lifestyle measures such as increased exercise and weight loss along with dietary modification. They should use simple analgesics for pain. There is no strong evidence supporting the use of antiinflammatory agents such as mesalazine or probiotics in this group. Those with a prior history of irritable bowel syndrome (IBS) or associated multiple somatic symptoms suggesting visceral hypersensitivity could reasonably be given a trial of proven IBS medications including antispasmodics or low-dose tricyclic antidepressants; however, there is not yet any trial evidence in diverticular disease to support this.

Patients with acute diverticulitis who do not show a marked septic response, and whose pain is manageable, are often treated at home with antibiotics by their primary care physician, although recent trials suggest this is unnecessary for most. Individuals who do not meet this criterion should be treated in hospital. The mainstay of treatment has been antibiotics and bowel rest. Recent randomized controlled trials (RCTs) have, however, shown that, in patients with computed tomography (CT)-confirmed uncomplicated acute diverticulitis, antibiotics can be safely omitted. In those with demonstrable abscesses, treatments include antibiotics, percutaneous drainage of abscesses or surgery for those with large abscesses (especially pelvic) and abscesses not amenable to drainage.

Patients with perforation are treated based on the symptoms and the extent of perforation. Those with minimal symptoms and localized extraluminal gas can be treated with antibiotics alone and close observation. However, those with more severe signs and symptoms should undergo surgery. There has been much interest in the use of laparoscopic lavage for the treatment of localized perforation with purulent peritonitis. However, recent RCTs have highlighted a greater reintervention rate in those treated with lavage, and the optimum management of these patients therefore remains unclear. Those in whom lavage is not appropriate or has failed require a laparotomy and resection of the affected segment with or without an anastomosis. In most patients, a Hartmann's procedure is performed with formation of a colostomy after resection and closure of the rectal stump. 
Prognosis and explanation to the patient

Individuals hospitalized with their first attack of acute diverticulitis have a risk of recurrence of around 1 in 4 over the next decade. Second and subsequent attacks have a risk of developing into complicated diverticular disease similar to that of the first attack, i.e. around 1 in 10 . Surgical treatment can be required for complications during the first admission in up to 1 in 10 patients. Elective surgery rates after the first attack are $<5 \%$ in the first year.

\section{Prevention}

Recurrence of acute diverticulitis is relatively uncommon, so studies of preventive treatments need to be prolonged and include large numbers. Two large high-quality trials of the anti-inflammatory drug mesalazine showed no reduction in recurrence rate. Rifaximin and probiotics have also been suggested as potential treatments, but the evidence that any treatment works for chronic symptomatic uncomplicated diverticular disease is weak. Such trials inevitably contain a mixture of patients who have IBS with coincidental diverticulosis and patients whose symptoms are caused by diverticulosis. Unfortunately, there is no biomarker that can objectively distinguish these patients, although a long history of IBS preceding the likely development of diverticulosis is helpful.

Many healthcare professionals advocate a high-fibre diet, and recent studies suggest that patients with diverticular disease tend to have a higher fibre intake. Although this often softens stools, there are no high-quality RCTs to prove this is beneficial. Indeed, some patients find it exacerbates symptoms of flatulence and bloating. 
Figure 1 Computed tomography scan showing acute diverticulitis with localised perforation (white arrow)

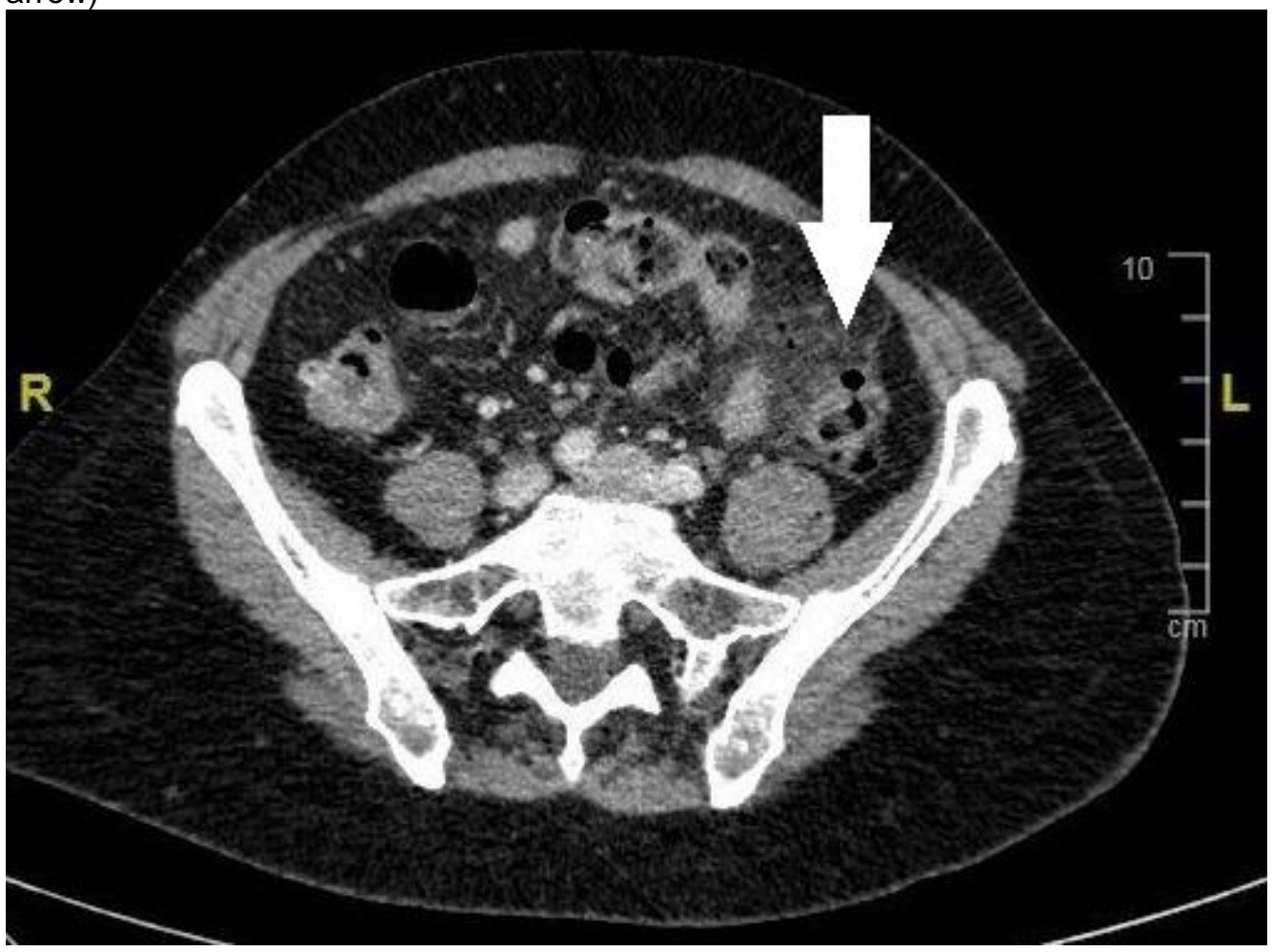


Table 1 Different diagnosis of diverticular disease

\begin{tabular}{|c|c|c|}
\hline System & Diagnoses & Key distinguishing features \\
\hline \multirow[t]{9}{*}{ Gastrointestinal } & Acute diverticulitis & Fever, well-localized pain \\
\hline & IBS & $\begin{array}{l}\text { Absence of inflammatory } \\
\text { markers, diffuse pain, } \\
\text { chronicity }\end{array}$ \\
\hline & Ischaemic bowel & Rectal bleeding \\
\hline & Omental infarction & \\
\hline & Epiploic appendagitis & \\
\hline & Volvulus & $\begin{array}{l}\text { Distended abdomen, loud } \\
\text { bowel sounds }\end{array}$ \\
\hline & Inflammatory bowel disease & $\begin{array}{l}\text { Rectal bleeding, elevated } \\
\text { inflammatory markers }\end{array}$ \\
\hline & Malignancy & $\begin{array}{l}\text { Progressive history, abdominal } \\
\text { mass }\end{array}$ \\
\hline & Infective colitis & Diarrhoea, fever \\
\hline \multirow[t]{3}{*}{ Genitourinary } & Urinary tract infection & $\begin{array}{l}\text { High fever, renal angle } \\
\text { tenderness }\end{array}$ \\
\hline & Renal colic & \\
\hline & Prostatitis & Disturbed micturition \\
\hline \multirow[t]{3}{*}{ Gynaecological } & Ovarian cysts & \\
\hline & Ectopic pregnancy & \\
\hline & Salpingitis & \\
\hline Miscellaneous & $\begin{array}{l}\text { Hernia } \\
\text { Psoas abscess } \\
\text { Retroperitoneal haemorrhage }\end{array}$ & \\
\hline
\end{tabular}

\section{Key References}

1. Humes D, Spiller RC. Colonic diverticular disease: medical treatments for acute diverticulitis. BMJ Clin Evid 2016; 2016. pii: 0405.

2. Marshall JR, Buchwald PL, Gandhi J, et al. Laparoscopic lavage in the management of Hinchey grade III diverticulitis: a systematic review. Ann Surg 2017; 265: 670-6.

3. Stollman N, Smalley W, Hirano I, et al. American Gastroenterological Association Institute guideline on the management of acute diverticulitis. Gastroenterology 2015; 149: 1944-9.

4. Regenbogen SE, Hardiman KM, Hendren S, et al. Surgery for diverticulitis in the 21st century: a systematic review. JAMA Surg 2014; 149: 292-303.

5. Morris AM, Regenbogen SE, Hardiman KM, et al. Sigmoid diverticulitis: a systematic review. JAMA 2014; 311: 287-97. 


\section{TEST YOURSELF}

To test your knowledge based on the article you have just read, please complete the questions below. The answers can be found at the end of the issue or online here.

\section{Question 1}

A 60-year-old man presented with severe left iliac fossa pain and swinging fevers.

On clinical examination, his temperature was $38.5^{\circ} \mathrm{C}$, heart rate 105 beats/minute, and blood pressure $135 / 78 \mathrm{mmHg}$. There was marked tenderness in the right iliac fossa.

What is the most appropriate investigation to confirm the likely diagnosis?
A. Full blood count
B. MRI
C. Flexible sigmoidoscopy
D. Contrast CT scan of the abdomen
E. Ultrasound

Correct answer: D. A CT scan with contrast allows the diagnosis of acute diverticulitis or its complications along with other pathology an alternative would be a non-contrast scan in those $B / D$ ) with renal failure. A MRI (B) or ultrasound (E) provides some information and can be used in patients in whom CT is contraindicated. Flexible sigmoidoscopy $(C)$ in the acute setting risk colonic perforation. A full blood count $(\mathrm{A})$ is useful in assessment as a marker for anaemia or sepsis but will not lead to a diagnosis of acute diverticulitis.

\section{Question 2}

A 70-year-old woman presented with a 3-day history of localized left iliac fossa pain.

On clinical examination, she appeared well. Her temperature was $37.5^{\circ} \mathrm{C}$, heart rate 94 beats $/$ minute, and blood pressure 130/75 $\mathrm{mmHg}$. There was localized tenderness in the right iliac fossa.

\section{Investigation}

-CT scan of the abdomen showed localized locules of free gas and inflammatory stranding consistent with a localized perforation

\section{What is likely to be the best treatment option?}

A. Immediate surgery with a Hartmann's procedure

B. Immediate surgery with laparoscopic lavage

C. Intravenous antibiotics and close observation

D. Immediate surgery with a primary anastomosis

E. Discharge

Correct answer: C. This woman is clinically well with a localized perforation. A trial of conservative management can be pursued as long as the patient is aware that any deterioration might lead to the need for surgery, which could include any of the surgical options above $(A, B, D)$. Discharging this patient would not be an option given the clinical findings (E).

\section{Question 3}

A 75 -year-old man presented with a 2-day history of left iliac fossa pain.

On clinical examination, he appeared unwell. His temperature was $38.5^{\circ} \mathrm{C}$, heart rate 100 beats/minute and blood pressure $132 / 65 \mathrm{mmHg}$.

\section{Investigations}

-C-reactive protein $150 \mathrm{mg} /$ litre $(<10)$

-CT scan of the abdomen showed inflammatory stranding with a $5 \mathrm{~cm}$ paracolonic abscess

\section{What is likely to be the best treatment option?}
A. Laparoscopic lavage
B. Immediate surgery with a Hartmann's procedure 
C. Intravenous antibiotics and close observation

D. Immediate surgery with a primary anastomosis

E. Percutaneous drainage and intravenous antibiotics

Correct answer: E. In a patient with a diverticular abscess, percutaneous drainage in the first instance if the abscess is amenable to this can prevent the need for emergency resectional surgery. Therefore this should be the first approach if the patient condition allows and local expertise in percutaneous drainage is available. Surgery could be considered if the abscess is large, situated in the pelvis. The choice of surgical approach depends on surgical expertise along with the patients condition and prior comorbidity and options include resection with or without anastomosis (B/D). Abscesses less than $2 \mathrm{~cm}$ can be treated by antibiotics alone $(C)$. Laparoscopic lavage is generally reserved for patients with purulent peritonitis rather than for the drainage of abscesses $(A)$. 